\title{
Inverse Square Lévy Walks are not Optimal Search Strategies for $d \geq 2$
}

\author{
Nicolas Levernier, ${ }^{1,2}$ Johannes Textor $\odot,^{3,4}$ Olivier Bénichou, ${ }^{5}$ and Raphaël Voituriez ${ }^{5,6}$ \\ ${ }^{1}$ Department of Theoretical Physics, University of Geneva, 1211 Geneva 4, Switzerland \\ ${ }^{2}$ Department of Biochemistry, University of Geneva, 1211 Geneva 4, Switzerland \\ ${ }^{3}$ Radboud Institute for Molecular Life Sciences, Radboud University Medical Centre, 6525 EZ Nijmegen, Netherlands \\ ${ }^{4}$ Institute for Computing and Information Sciences, Radboud University, 6525 EZ Nijmegen, Netherlands \\ ${ }^{5}$ Laboratoire de Physique Théorique de la Matière Condensée, UMR 7600 CNRS/UPMC, 4 Place Jussieu, 75255 Paris Cedex, France \\ ${ }^{6}$ Laboratoire Jean Perrin, UMR 8237 CNRS/UPMC, 4 Place Jussieu, 75255 Paris Cedex, France
}

(Received 24 September 2019; revised manuscript received 22 November 2019; accepted 30 January 2020; published 26 February 2020)

\begin{abstract}
The Lévy hypothesis states that inverse square Lévy walks are optimal search strategies because they maximize the encounter rate with sparse, randomly distributed, replenishable targets. It has served as a theoretical basis to interpret a wealth of experimental data at various scales, from molecular motors to animals looking for resources, putting forward the conclusion that many living organisms perform Lévy walks to explore space because of their optimal efficiency. Here we provide analytically the dependence on target density of the encounter rate of Lévy walks for any space dimension $d$; in particular, this scaling is shown to be independent of the Lévy exponent $\alpha$ for the biologically relevant case $d \geq 2$, which proves that the founding result of the Lévy hypothesis is incorrect. As a consequence, we show that optimizing the encounter rate with respect to $\alpha$ is irrelevant: it does not change the scaling with density and can lead virtually to any optimal value of $\alpha$ depending on system dependent modeling choices. The conclusion that observed inverse square Lévy patterns are the result of a common selection process based purely on the kinetics of the search behavior is therefore unfounded.
\end{abstract}

DOI: 10.1103/PhysRevLett.124.080601

Lévy walks [1] were introduced as a minimal random walk model that displays a superdiffusive scaling, while preserving a finite speed, and were originally motivated by various physical processes such as phase diffusion in Josephson junctions [2,3] or passive diffusion in turbulent flow fields [4]. Shlesinger and Klafter [5] were the first to report that, due to their weak oversampling properties, Lévy walks provide a more efficient way to explore space than normal random walks. This observation led Viswanathan et al. [6,7] to propose the following Lévy search model (Fig. 1): they consider a searcher that performs ballistic flights of uniformly distributed random directions and constant speed, whose lengths $l$ are drawn from a distribution with power law tails $p(l) \sim C s^{\alpha} / l^{1+\alpha}$ $(l \rightarrow \infty)$ characterized by the Lévy exponent $\alpha \in[0,2]$, where $s$ is a scale parameter and $C$ a dimensionless normalization constant. The authors of Ref. [7] consider an infinite space of dimension $d$ with Poisson distributed (i.e., with uniform density) immobile targets of density $\rho$, which are captured as soon as within a detection distance $a$ from the searcher. Two alternative hypotheses that lead to two very different optimal strategies (i.e., strategies maximizing the capture rate $\eta=\lim _{t \rightarrow \infty} n_{t} / t$ with respect to $\alpha$, where $n_{t}$ is the mean number of targets detected at time $t$ ) are studied. (a) In the first case of "revisitable targets", meaning that, as soon as detected, a target reappears and

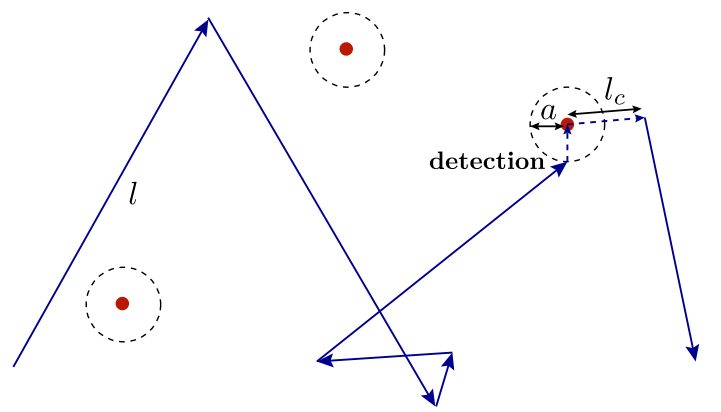

FIG. 1. The Lévy walk search model and its parameters. We consider a slightly more general version of the model originally introduced in Ref. [7], here in $d=2$. The pointlike searcher performs ballistic flights at constant speed $v$ (that can be set to 1 ) in uniformly distributed random directions. The length $l$ of each flight is drawn from a distribution which satisfies $p(l) \sim C s^{\alpha} / l^{1+\alpha}$. In numerical simulations we used $p(l)=(2 \pi)^{-1} l \int e^{i k l \cos \theta} e^{-s^{\alpha} k^{\alpha}} k d k d \theta$ for $d=2$. Targets are immobile and uniformly distributed in infinite space with density $\rho$. A target is captured as soon as located within the detection radius $a$ (that can be set to 1) of the searcher, and is regenerated immediately after detection. To avoid systematic recapture of the same target, an arbitrary rule is required, such as a cutoff time $\tau_{c}>a / v$ before target regeneration, or a cutoff distance from the target $l_{c}>a$ (which is the prescription that we used in numerical simulations) from which the walk is restarted. Finally, the model in its minimal form involves the following parameters: $a$ (that defines the unit length), $v$ (that defines the unit time), the target density $\rho$, the Lévy exponent $\alpha$ and scale $s$ necessary to define $p(l)$, and the cutoff length $l_{c}$ (or equivalently a cutoff time $\tau_{c}$ ). 
stays immobile at the same location, the authors claim that in the small density limit the encounter rate is optimized for a Lévy exponent $\alpha \rightarrow 1$, the so called inverse square Lévy walk, and independently of the small scale characteristics of $p(l)$ or space dimension $d$. (b) In the second case of "destructive search" where each target can be found only once, the optimal strategy is not of Lévy type, but reduces to a simple linear ballistic motion for all $d$.

The optimality of inverse square Lévy walks claimed in Ref. [7] is at the core of the Lévy hypothesis, which has been the reference theoretical framework for the analysis of trajectories of broad classes of living systems, from molecular motors [8] to cells [9] and foraging animals [6,7,10-13]; many studies have indeed interpreted field data as Lévy walks, thereby concluding that their observation was the result of a selection process based on the optimality claimed in Ref. [7]. In fact, since then the relevance to field data of the condition (a) of revisitable targets has been questioned [14-17], and the identification of Lévy patterns from real data has been debated $[18,19]$. On the theoretical side, several alternative models, or variations of the original model [7] have been proposed [20-23]. By allowing for more degrees of freedom, or by modifying the hypothesis of the original optimization problem, these were shown to potentially lead to different optimal strategies. However, so far all studies acknowledged the original result [7] as a founding benchmark in the field, and none has contested its technical validity.

In this Letter, we show on the basis of the same model that while the original analytical expression of the encounter rate with targets for Lévy walks proposed in Ref. [7] is correct in space dimension $d=1$, it is incorrect for $d \geq 2$. As a consequence, the conclusion that inverse square Lévy walks are optimal search strategies is not valid in the biologically relevant case $d \geq 2$. In fact, relying on a recently developed framework to analyze non-Markovian target search processes such as Lévy walks [24,25], we show that, as opposed to what is claimed in Ref. [7], for $d \geq 2$ the encounter rate of Lévy walks with sparse Poisson distributed targets (i) displays a linear dependence on the concentration of targets for all values of the Lévy exponent, and (ii) can therefore be only marginally maximized, and for a broad range of values of the Lévy exponent controlled by model dependent parameters, which makes the optimization nonuniversal. This invalidates the claim that inverse square Lévy walks are optimal search strategies for $d \geq 2$, and more generally makes the optimization of Lévy search processes with respect to the Lévy exponent nonrobust and thus irrelevant biologically for $d \geq 2$. The conclusion that observed inverse square Lévy patterns across very different systems are the result of a common selection process based purely on the kinetics of the search behavior is therefore unfounded.

Technically, it is straightforward to show that for the case (b) of destructive search the optimal search strategy is achieved for $\alpha \rightarrow 0$ (straight ballistic motion), as stated in Ref. [7]; the ballistic strategy indeed minimizes oversampling of space, as discussed in Ref. [5]. This intuitive argument however fails in the case (a) of revisitable targets, which we discuss from now on. Let us first note that $1 / \eta \equiv T$ is the mean time elapsed between successive capture events or in other words the mean first-passage time (MFPT) to any target for a searcher that starts immediately after a capture event. While the determination of MFPTs of random walks has been studied at length in the literature because of the relevance of this observable to various fields [26-30], its analytical calculation for non-Markovian random walks, such as Lévy walks, has remained until recently a technical challenge. For that reason, the analytical determination of $\eta$ proposed originally in Ref. [7] involved uncontrolled hypotheses, and this result was proved correct analytically later in Ref. [31], but only for $d=1$. Of note, in Ref. [7] the predicted scaling of $\eta$ with target density was supported by numerical simulations, but again only for $d=1$; in $d=2$, numerical simulations were shown for a single value of the density, thereby precluding any comparison with the predicted scaling.

Recently, new techniques have been introduced to determine analytically the MFPT of non-Markovian random walks to a single target in a confining volume $V$ in any space dimension $d$ in the large $V$ limit, first in the case of Gaussian processes [24], and lately for general scale-invariant processes [25]. Following a classical mean-field type argument [17,32], which was validated numerically, this result also yields in the large $V$ limit the MFPT to any target in infinite space with a concentration of Poisson distributed targets $\rho \equiv 1 / V$, which is precisely the quantity that we aim at computing. We here apply these techniques to Lévy walks, which, importantly, requires us to treat separately the cases of compact and noncompact exploration.

In the case of a compact walk, which occurs for Lévy walks for $d=1$, it is found [25] that the MFPT is given by

$$
T \underset{V \rightarrow \infty}{\sim} A V^{d_{w}(1-\theta) / d} l_{c} d_{w} \theta
$$

where $A$ is a numerical constant, $d_{w}$ the walk dimension, $\theta$ the persistence exponent, and $l_{c}$ the cutoff length introduced in Fig. 1. The walk dimension is given by $d_{w}=1$ for $\alpha<1$, and $d_{w}=\alpha$ for $\alpha>1$, and the persistence exponent is $\theta=\alpha / 2$ for $\alpha<1$ and $\theta=1 / 2$ for $\alpha>1$ [25]. Thus, we get

$$
T \underset{V \rightarrow \infty}{\sim} \begin{cases}D_{1}(\alpha) V^{1-\alpha / 2} & (\alpha<1) \\ D_{1}(\alpha) V^{\alpha / 2} & (\alpha>1)\end{cases}
$$

where $D_{1}$ is a numerical constant independent of the volume $V$.

In the case of noncompact random walks, which is the case of Lévy walks for $d \geq 2$, the MFPT satisfies [25] 


$$
\underset{V \rightarrow \infty}{\sim} A \frac{V^{\left(d_{w}+\psi\right) / d}}{a^{\psi}}\left[1-B\left(\frac{a}{l_{c}}\right)^{\psi /}\right]
$$

In the latter, $A$ and $B$ are numerical constants, $d_{w}$ denotes the walk dimension, and $\psi$ the transience exponent. Their corresponding values for a Lévy walk of parameter $\alpha$ is given by $d_{w}=1$ and $\psi=d-1$ for $\alpha<1$, and $d_{w}=\alpha$ and $\psi=d-\alpha$ for $\alpha>1$ [25]. Hence, for any $\alpha \in[0,2]$, we get

$$
T \underset{V \rightarrow \infty}{\sim} D_{d}\left(\alpha, l_{c} / a\right) V
$$

where $D_{d}\left(\alpha, l_{c} / a\right)$ is a numerical constant depending on $\alpha$, $d$ and the microscopic parameters $l_{c}$ and $a$, but not on the volume $V$.

Using Eqs. (4) and (2) with $\rho=1 / V$, we finally obtain analytically the mean capture rate $\eta$, thereby solving explicitly the original problem introduced in Ref. [7] and recalled above (Fig. 1). It is found that

$$
\eta(\rho) \underset{\rho \rightarrow 0}{\sim}\left\{\begin{array}{llll}
K_{1}(\alpha) \rho^{1-\alpha / 2} & d=1 & \text { and } & 0<\alpha<1 \\
K_{1}(\alpha) \rho^{\alpha / 2} & d=1 & \text { and } & 1<\alpha<2 \\
K_{d}(\alpha) \rho & d \geq 2 & \text { and } & 0<\alpha<2
\end{array},\right.
$$

where the constants $K_{d}$ are independent of $\rho$, but depend on the cutoff length $l_{c}$ that characterizes the condition of restart after a capture event and the scale parameter $s$ that enters the definition of $p(l)$ (see Fig. 1; this formulation contains in particular the original model as introduced in Ref. [7]). This result is valid asymptotically in the relevant limit of sparse targets $(\rho \rightarrow 0)$, and was checked numerically in Figs. 2 and 3.

Several comments are in order. (i) For $d=1$, the result of Eq. (5) is consistent with the original result for $\eta(\rho)$ given in Ref. [7], as confirmed numerically in Fig. 2(b) and analytically in Ref. [31]; in particular, in the $\rho \rightarrow 0$ limit, $\eta$ is maximized for the inverse square Lévy walk $\alpha=1$, as claimed in Ref. [7]. Of note, this optimum is robust in the sense that the gain $\eta_{\max } / \eta$ is arbitrarily large in the limit $\rho \rightarrow 0$ for all values of the parameters $s$ and $l_{c}$, and is therefore critically controlled by the parameter $\alpha$ only [Fig. 3(c)]. (ii) However, for the biologically relevant case $d \geq 2$ the prediction for $\eta(\rho)$ given in Ref. [7], claimed to be identical to the $d=1$ case, is incorrect. Indeed, the result of Eq. (5) shows that $\eta$ depends linearly on $\rho$ for all $\alpha$ in contrast to the $d=1$ case [confirmed numerically in Fig. 2(a)]. (iii) This has strong consequences on the maximization of $\eta$. In fact, for $d \geq 2$, the dependence of $\eta$ on $\alpha$ lies only in the prefactor $K_{d}$. This implies first that the gain $\eta_{\max } / \eta$ achieved by varying $\alpha$ is bounded even in the limit $\rho \rightarrow 0$. In other words, tuning $\alpha$ can only yield a marginal gain, and therefore does not present a decisive selective advantage, as opposed to the $d=1$ case [Figs. 3(a) and 3(b)]. Second, as we show numerically [Fig. 3(a)], $K_{d}(\alpha)$ presents bounded variations that depend drastically on the choice of parameters $s$ and $l_{c}$, which could be arbitrary depending on the system studied. In particular, by performing minute variations of $s$ and $l_{c}$ it is
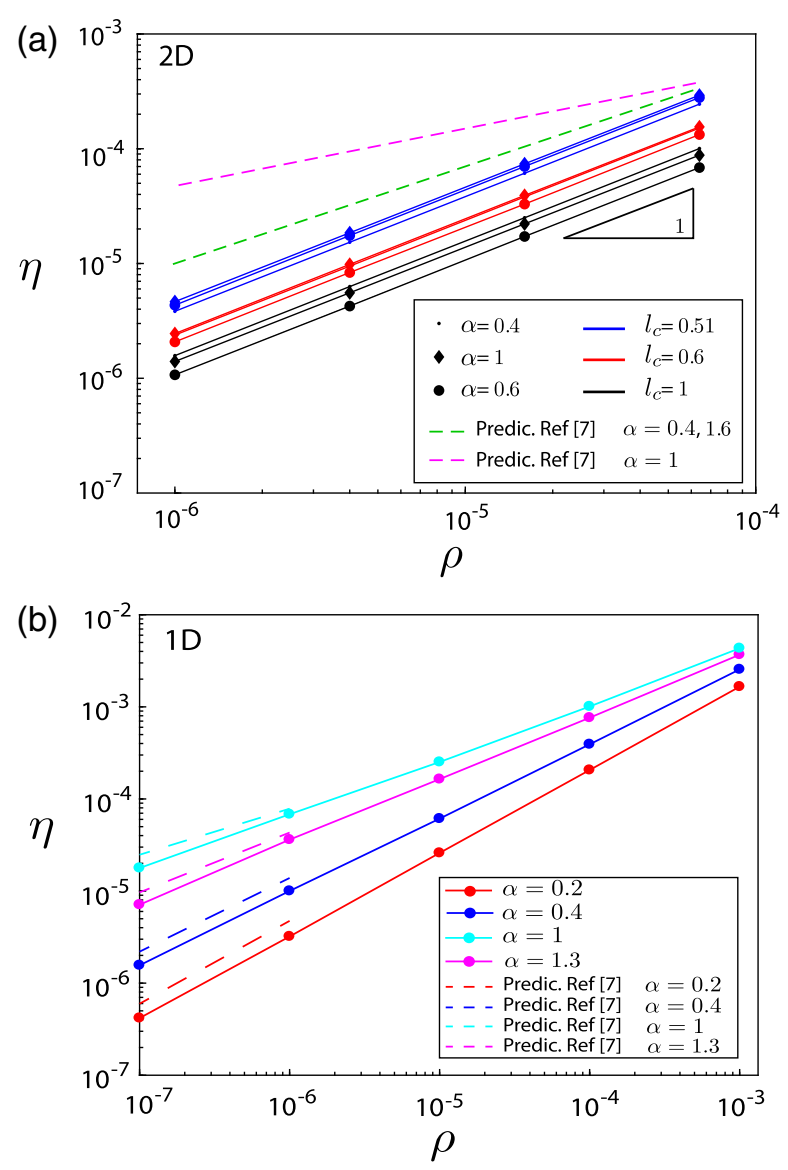

FIG. 2. The capture rate of Lévy walkers has different scalings with target density for $d=2$ and $d=1$. Capture rate $\eta$ as a function of target density ( $\rho$ ) for different values of $\alpha$. (a) Case $d=2$. Simulations are performed with 4000 Poisson-distributed targets of detection radius $a=0.5$ in a $2 d$ square box of linear size $200 / \sqrt{\rho}$ with periodic boundary conditions, following the dynamics defined in Fig. 1 with $s=0.1$. Upon each detection event, the searcher stops and restarts immediately from a distance $l_{c}$ from the target. In all cases, it is found that $\eta$ grows linearly with $\rho$ as predicted by Eq. (5). Numerical simulations (symbols and plain lines) are compared to the predicted scaling of Ref. [7] (dashed lines), and to our linear prediction (slope 1). (b) Case $d=1$. Simulations are performed with Poisson distributed targets and make use of the dynamics defined in Ref. [7]. The jump distribution is a truncated Pareto law: $p(l)=C / l^{1+\alpha}$ for $l>l_{c}$ and $p(l)=0$ for $l<l_{c}$, where $C$ is a normalization constant; here $l_{c}=a=1$. Numerical simulations (symbols and plain lines) are compared to the predicted scaling of [7] (dashed lines) that we recover in this Letter.

found that $\eta$ can be maximised for a broad range of values of $\alpha \in$ [0,2], (Fig. 3). This overall makes the optimization with respect to $\alpha$ biologically irrelevant for $d \geq 2$, and in particular invalidates the optimality of inverse square Lévy walks claimed in Ref. [7] for generic values of $s$ and $l_{c}$.

These theoretical results have been fully validated by numerical simulations (Figs. 2 and 3), which confirm in particular the linear dependence of $\eta$ on $\rho$ independently of $\alpha$ for $d=2$ as predicted by Eq. (2) [Fig. 2(a)], and the 

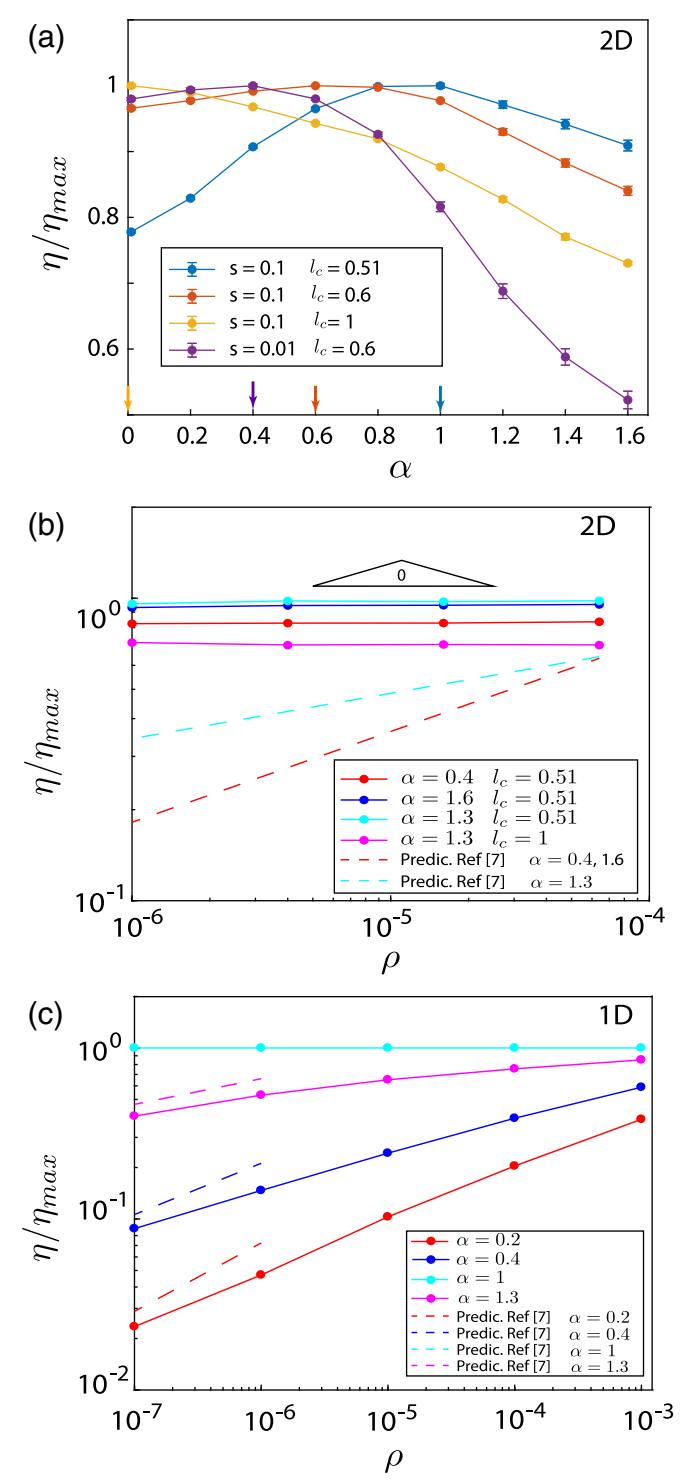

FIG. 3. A broad range of values of the Lévy exponent $\alpha$ can optimize the capture rate for $d=2$. (a) Normalized capture rate as a function of $\alpha$ for different values of the cutoff distance $l_{c}$ and scale parameter $s$ for $d=2$. Simulations (symbols and lines) are performed with 4000 Poisson-distributed targets of detection radius $a=0.5$ in a $2 d$ box of size $1000 \times 200$ with periodic boundary conditions. The capture rate can be maximized for different values $\alpha \in[0,2]$ (arrows) depending on the choice of parameters $l_{c}$ and $s$. (b) Normalized capture rate as a function of $\rho$ for different values of $\alpha$ for $d=2$. Simulations (symbols and lines) are performed with 4000 Poisson-distributed targets of detection radius $a=0.5$ in $2 d$ boxes of various sizes, with periodic boundary conditions and agree with our prediction (independence on $\rho$, slope $0)$. Dashed lines show the prediction of Ref. [7], which is invalid for $d=2$. The gain is bounded and independent of the target density $\rho$ for $\rho \rightarrow 0$, as predicted by Eq. (5). (c) Normalized capture rate as a function of $\rho$ for different values of $\alpha$ for $d=1$ (same dynamics as in Fig. 2). Simulations (symbols and lines) are in agreement with the prediction of Eq. (5) (consistent with Ref. [7]) shown in dashed lines. The gain diverges in the limit $\rho \rightarrow 0$. Note however the slow convergence to the exact scaling when $\rho \rightarrow 0$. sensitivity of $K_{d}$ to the system dependent parameters $s$ and $l_{c}$ [Fig. 3(a)]. In the context of animal foraging, the diverging gain at low target density obtained for $d=1$ (which could be relevant to specific biological examples) means that the implied optimal foraging behavior at $\alpha=1$ is expected to be a robust property that does not depend on the small scale characteristics of the specific biological system under study. Conversely, for $d \geq 2$, which is the generic biologically relevant case, this conclusion does not hold because the optimal foraging behavior presents only a limited gain, and may change even if seemingly minor changes are made to the system. For example, very different optimal values of $\alpha$ can be obtained simply by allowing the searcher to have a short-term memory that would modify the small scale features of $p(l)$ or the way in which the first step after finding a target is performed [Fig. 3(a)]. In fact, we found that $\alpha=1$ optimizes the encounter rate for $d=2$ only in the specific regime $l_{c} \rightarrow a$ and $s \ll a$, for which the problem is indeed expected to be effectively amenable to $d=1$ [see Fig. 3(a)]. Of note, for $d \geq 2$ it is found numerically that $\eta$ seems to be always maximized for $\alpha<2$, i.e., away from the Brownian limit, thereby suggesting that in this model Lévy walks are more efficient than Brownian walks. However, we stress again that the scaling of $\eta$ with target density is unchanged (up to logarithmic corrections for $d=2$ ), which makes the optimization overall of marginal importance.

Altogether, this shows that inverse square Lévy walks are not generic optimal search strategies for $d \geq 2$, and therefore that the conclusion found in many studies that observed inverse square Lévy patterns are the result of a selection process is unfounded. Importantly, we stress first that these results do not invalidate the original idea that Lévy walks can be efficient to explore space [5], but disprove the specific role of inverse square Lévy walks and their optimality for $d \geq 2$. Second, on the experimental side, these results do not question the validity of observations of power-law-like patterns in field data, but refute the classical hypothesis that the observation of inverse square Lévy walks would be the result of a selection process based on the kinetics of the search behavior. Alternatively the observed patterns could be the result of various environmental parameters, such as the spatial distribution of prey, as suggested in Ref. [12] and observed in Ref. [33].

This work was supported by ERC Grant No. FPTOpt277998.

[1] V. Zaburdaev, S. Denisov, and J. Klafter, Rev. Mod. Phys. 87, 483 (2015).

[2] T. Geisel, J. Nierwetberg, and A. Zacherl, Phys. Rev. Lett. 54, 616 (1985).

[3] M. F. Shlesinger and J. Klafter, Phys. Rev. Lett. 54, 2551 (1985). 
[4] M. F. Shlesinger, B. J. West, and J. Klafter, Phys. Rev. Lett. 58, 1100 (1987).

[5] M. F. Shlesinger and J. Klafter, Lévy Walks vs Lévy Flights (H. E. Stanley and N. Ostrowski, Dordrecht, 1986).

[6] G. M. Viswanathan, V. Afanasyev, S. V. Buldyrev, E. J. Murphy, P. A. Prince, and H. E. Stanley, Nature (London) 381, 413 (1996).

[7] G. M. Viswanathan, S. V. Buldyrev, S. Havlin, M. G. E. da Luz, E. P. Raposo, and H. E. Stanley, Nature (London) 401, 911 (1999).

[8] K. Chen, B. Wang, and S. Granick, Nat. Mater. 14, 589 (2015).

[9] T. H. Harris, E. J. Banigan, D. A. Christian, C. Konradt, E. D. Tait Wojno, K. Norose, E. H. Wilson, B. John, W. Weninger, A. D. Luster et al., Nature (London) 486, 545 (2012).

[10] O. Miramontes, O. DeSouza, L. R. Paiva, A. Marins, and S. Orozco, PLoS One 9, e111183 (2014).

[11] D. W. Sims, E. J. Southall, N. E. Humphries, G. C. Hays, C. J. A. Bradshaw, J. W. Pitchford, A. James, M. Z. Ahmed, A. S. Brierley, M. A. Hindell et al., Nature (London) 451, 1098 (2008).

[12] N. E. Humphries, N. Queiroz, J. R. M. Dyer, N. G. Pade, M. K. Musyl, K. M. Schaefer, D. W. Fuller, J. M. Brunnschweiler, T. K. Doyle, J. D. R. Houghton et al., Nature (London) 465, 1066 (2010).

[13] N. E. Humphries, H. Weimerskirch, N. Queiroz, E.J. Southall, and D. W. Sims, Proc. Natl. Acad. Sci. U.S.A. 109, 7169 (2012).

[14] O. Benichou, C. Loverdo, M. Moreau, and R. Voituriez, Phys. Rev. E 74, 020102(R) (2006).

[15] O. Bénichou, M. Coppey, M. Moreau, and R. Voituriez, Europhys. Lett. 75, 349 (2006).

[16] V. Tejedor, R. Voituriez, and O. Bénichou, Phys. Rev. Lett. 108, 088103 (2012).
[17] O. Bénichou, C. Loverdo, M. Moreau, and R. Voituriez, Rev. Mod. Phys. 83, 81 (2011).

[18] A. M. Edwards, R. A. Phillips, N. W. Watkins, M. P. Freeman, E. J. Murphy, V. Afanasyev, S. V. Buldyrev, M. G. E. da Luz, E. P. Raposo, H. E. Stanley et al., Nature (London) 449, 1044 (2007).

[19] S. Benhamou, Ecology 88, 1962 (2007).

[20] F. Bartumeus, D. Campos, W. S. Ryu, R. Lloret-Cabot, V. Méndez, and J. Catalan, Ecol. Lett. 19, 1299 (2016).

[21] D. Campos, F. Bartumeus, E. P. Raposo, and V. Méndez, Phys. Rev. E 92, 052702 (2015).

[22] F. Bartumeus, E. P. Raposo, G. M. Viswanathan, and M. G. E. da Luz, PLoS One 9, e106373 (2014).

[23] M. E. Wosniack, M. C. Santos, E. P. Raposo, G. M. Viswanathan, and M. G. E. da Luz, PLoS Comput. Biol. 13, e1005774 (2017).

[24] T. Guérin, N. Levernier, O. Bénichou, and R. Voituriez, Nature (London) 534, 356 (2016).

[25] N. Levernier, O. Bénichou, T. Guérin, and R. Voituriez, Phys. Rev. E 98, 022125 (2018).

[26] S. Redner, A Guide to First-Passage Processes (Cambridge University Press, Cambridge, England, 2001).

[27] M. F. Shlesinger, Nature (London) 443, 281 (2006).

[28] S. Condamin, O. Bénichou, V. Tejedor, R. Voituriez, and J. Klafter, Nature (London) 450, 77 (2007).

[29] M. F. Shlesinger, Nature (London) 450, 40 (2007).

[30] R. Metzler, G. Oshanin, and S. Redner, First Passage Problems: Recent Advances (World Scientific, Singapore, 2014).

[31] S. V. Buldyrev, S. Havlin, A. Y. Kazakov, M. G. E. da Luz, E. P. Raposo, H. E. Stanley, and G. M. Viswanathan, Phys. Rev. E 64, 041108 (2001).

[32] O. Berg and C. Blomberg, Biophys. Chem. 4, 367 (1976).

[33] D. Boyer, G. Ramos-Fernández, O. Miramontes, J. Mateos, G. Cocho, H. Larralde, H. Ramos, and F. Rojas, Proc. Biol. Sci. 273, 1743 (2006). 